\title{
Mortality and Readmission Rates After Heart Failure: A Systematic Review and Meta-Analysis
}

\author{
Tian $\operatorname{Lan}^{1,2, *}$ \\ Yan-Hui Liao ${ }^{3, *}$ \\ Jian Zhang ${ }^{2}$ \\ Zhi-Ping Yang ${ }^{4}$ \\ Gao-Si Xu ${ }^{5}$ \\ Liang Zhu' \\ Dai-Ming Fan ${ }^{4}$ \\ 'Department of Health Care \\ Management and Medical Education, The \\ School of Military Preventive Medicine, \\ Air Force Medical University, Xi'an, \\ People's Republic of China; ${ }^{2}$ Department \\ of Health Care Management, The Second \\ Affiliated Hospital of Nanchang \\ University, Nanchang, People's Republic \\ of China; ${ }^{3}$ Department of Cardiology, \\ The Second Affiliated Hospital of \\ Nanchang University, Nanchang, People's \\ Republic of China; ${ }^{4}$ State Key Laboratory \\ of Cancer Biology and National Clinical \\ Research Center for Digestive Diseases, \\ Xijing Hospital of Digestive Diseases, Air \\ Force Medical University, Xi'an, People's \\ Republic of China; ${ }^{5}$ Department of \\ Nephrology, The Second Affiliated \\ Hospital of Nanchang University, \\ Nanchang, People's Republic of China
}

*These authors contributed equally to this work
Correspondence: Liang Zhu; Dai-Ming Fan Email liangjulia0317@163.com; li2020I226@|63.com
Objective: The current work aimed to examine the rates of and risk factors for mortality and readmission after heart failure (HF).

Setting: A systematic search was carried out in PubMed, the Cochrane Library, and EMBASE to identify eligible reports. The random-effects model was utilized to evaluate the pooled results.

Participants: A total of 27 studies with 515,238 participants were finally meta-analysed. The HF patients had an average age of 76.3 years, with $51 \%$ of the sample being male, in the pooled analysis.

Primary and Secondary Outcome Measures: The outcome measures were 30-day and 1 -year readmission rates, mortality, and risk factors for readmission and mortality.

Results: The effect sizes for readmission and mortality were estimated as the mean and $95 \%$ confidence interval (CI). The estimated 30-day and 1-year all-cause readmission rates were 0.19 (95\% CI $0.14-0.23$ ) and 0.53 (95\% CI 0.46-0.59), respectively, while the all-cause mortality rates were 0.14 (95\% CI $0.10-0.18$ ) and 0.29 (95\% CI $0.25-0.33$ ), respectively. Comorbidities were highly prevalent in individuals with HF.

Conclusion: Heart failure hospitalization is followed by high readmission and mortality rates.

Keywords: heart failure, meta-analysis, prevalence, readmission, mortality, hospitalization

\section{Background}

Heart failure (HF) represents a global public health threat. The effect of HF on the elderly population is disproportionate. Even assuming that the incidence for a specific age, sex, or ethnicity is stable, heart failure prevalence shows a steady elevation over the next 20 years, ${ }^{1}$ mainly in association with population ageing. ${ }^{2}$ Epidemiological changes in usual risk factors for heart failure may influence the above prediction. Even if the incidence for a specific age, sex, or race remains stable, the prevalence rates of hypertension and coronary heart disease would rise due to demographic changes. ${ }^{3}$ Meanwhile, the incidence rates of obesity ${ }^{4}$ and diabetes $^{5}$ for specific ages, sexes, and races are also expected to increase, and the increased prevalence rates of these risk factors may further elevate the prevalence of HF. As a result, heart failure remains a substantial and growing public health burden. According to available data in Europe and the United States, ${ }^{2-6}$ the prevalence of heart failure ranges from $1 \%$ to $14 \%$. When all adults are included, heart failure is considered a chronic debilitating disease regardless of age. ${ }^{2,5}$

In other developed countries, the one-year mortality rate after hospitalization for $\mathrm{HF}$ is $25 \%-30 \%{ }^{7}$ which is higher than those of many common cancers. ${ }^{8}$ The 
prevalence of heart failure increases with age. ${ }^{9,10}$ In addition, heart failure is the main cause of hospitalization in individuals over 64 years of age. ${ }^{11}$ The treatment of heart failure is important for improving the prognosis of patients and reducing health system expenditures. ${ }^{12}$ In addition, the in-hospital mortality rate of HF patients is relatively low; however, high rates of death and readmission are found after discharge. $^{13}$

Readmission constitutes a common negative result for health care facilities and patients and a huge financial burden imposed on medical insurance beneficiaries and private payers. ${ }^{11}$ Decreased 30-day readmission and 30day hospital mortality rates are weak but significantly correlated. ${ }^{14}$ From 2001-2003 to 2009-2011, the 30-day readmission rate following myocardial infarction declined from $20.5 \%$ to $15.8 \%$, although the trend decreased slightly upon adjustment for patient features and treatments. ${ }^{15}$ Predicting the risk factors for and causes of 30-day rehospitalization would help optimize the allocation of meagre medical resources and design profitable and viable interventions. ${ }^{16,17}$

However, multiple previous trials have been conducted in single centres with few patients, with inconsistent readmission and mortality rates in heart failure patients. For instance, 30-day readmission rates for heart failure in previous reports ranged between $4.3 \%$ and $30.4 \%{ }^{18-28}$ Based on the above, further assessing the prevalence and potential causes of and risk factors for readmission is of prime importance. Therefore, this meta-analysis aimed to examine the prevalence of readmission after HF, as well as the potential risk factors for and causes of HF. In addition, we discussed potential intervention approaches for mitigating the risk of readmission after HF.

\section{Methods}

\section{Search Strategy}

Two independent medical librarians systematically searched three electronic databases, including PubMed,

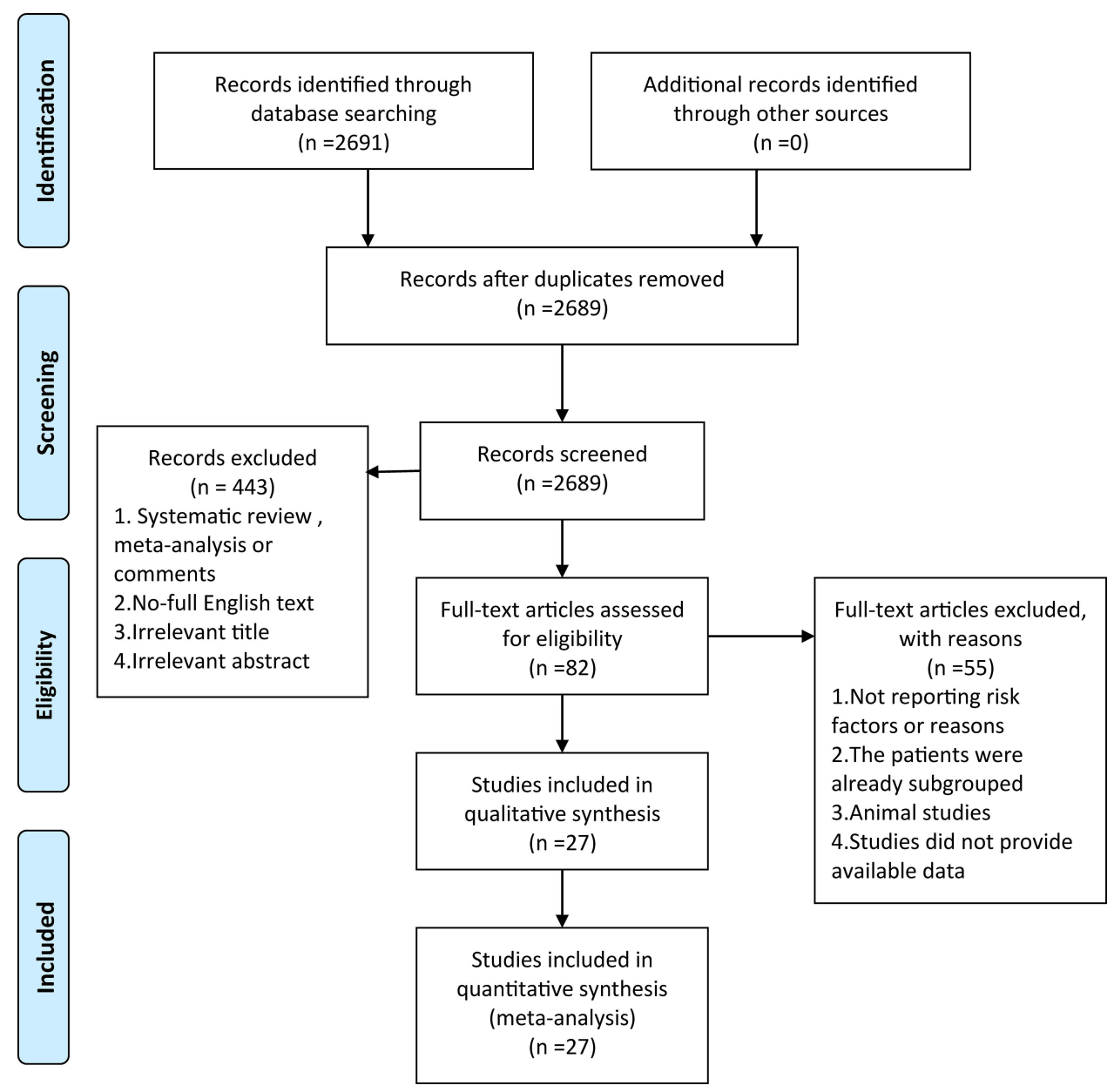

Figure I Flow diagram of the study. 
Table I Characteristics of Included Studies

\begin{tabular}{|c|c|c|c|c|c|c|}
\hline $\begin{array}{l}\text { Author } \\
\text { (Year) }\end{array}$ & Country & Study Period & $\begin{array}{l}\text { Method of HF } \\
\text { Diagnosis }\end{array}$ & Data Source & Study Type & $\begin{array}{l}\text { Study } \\
\text { Population }\end{array}$ \\
\hline $\begin{array}{l}\text { Aizawa } \\
\mathrm{H} 2015^{18}\end{array}$ & Japan & $\begin{array}{l}2012.4 .1- \\
2013.3 .31\end{array}$ & ICD-IO & DPC database & $\begin{array}{l}\text { Retrospective } \\
\text { cohort study }\end{array}$ & $\geq 15$ \\
\hline $\begin{array}{l}\text { Arenja } \\
\mathrm{N} 2011^{32}\end{array}$ & Switzerland & $\begin{array}{l}2001.5-2002.4 \\
2006.4-2007.3\end{array}$ & $\begin{array}{l}\text { Two } \\
\text { independent } \\
\text { cardiologists }\end{array}$ & University Hospital of Basel & Prospective study & - \\
\hline $\begin{array}{l}\text { Babayan ZV } \\
2003^{33}\end{array}$ & USA & $\begin{array}{l}\mid 996.1 .1- \\
\mid 997.12 .31\end{array}$ & $\begin{array}{l}\text { Modified } \\
\text { Framingham } \\
\text { criteria }\end{array}$ & Johns Hopkins Hospital & $\begin{array}{l}\text { Retrospective } \\
\text { cohort }\end{array}$ & - \\
\hline $\begin{array}{l}\text { Bradford } \\
\text { C } 2016^{19}\end{array}$ & USA & $\begin{array}{l}2008.10- \\
2014.11\end{array}$ & ICD-9-CM & Sharp Memorial Hospital & $\begin{array}{l}\text { Retrospective } \\
\text { observational } \\
\text { study }\end{array}$ & - \\
\hline $\begin{array}{l}\text { Chaudhry SI } \\
2010^{34}\end{array}$ & USA & $\begin{array}{l}\text { |998.4-1999.3, } \\
2000.7-200 \mid .6\end{array}$ & ICD-9-CM & Medicare & - & - \\
\hline $\begin{array}{l}\text { Choi DJ } \\
2011^{35}\end{array}$ & Korea & $2004.6-2009.4$ & $\begin{array}{l}\text { Framingham } \\
\text { criteria }\end{array}$ & KorHF Registry database & - & - \\
\hline $\begin{array}{l}\text { Coles AH } \\
2015^{36}\end{array}$ & USA & $\begin{array}{l}1995,2000, \\
2002,2004, \\
2006\end{array}$ & $\begin{array}{l}\text { Framingham } \\
\text { criteria, ICD-9 }\end{array}$ & Massachusetts medical centers & - & - \\
\hline $\begin{array}{l}\text { Corrao } \\
\text { G } 2015^{20}\end{array}$ & Italy & 2011 & ICD-9 & HCU Databases & $\begin{array}{l}\text { Retrospective } \\
\text { cohort study }\end{array}$ & $\geq 50$ \\
\hline $\begin{array}{l}\text { Costa } \\
\text { D } 2018^{37}\end{array}$ & Argentina & $\begin{array}{l}2016.6 .1- \\
2017.5 .31\end{array}$ & $\begin{array}{l}\text { Framingham } \\
\text { criteria }\end{array}$ & University Hospital in Buenos Aires & $\begin{array}{l}\text { Prospective, } \\
\text { observational } \\
\text { study }\end{array}$ & - \\
\hline Dai S $2016^{21}$ & USA & - & - & Florida Hospital & Prospective study & $20 \sim 89$ \\
\hline $\begin{array}{l}\text { Eapen ZJ } \\
2013^{38}\end{array}$ & USA & $2005.1-2009.12$ & ICD-9 & CMS & - & $\geq 65$ \\
\hline $\begin{array}{l}\text { Fernandez- } \\
\text { Gasso } \\
\text { L } 2017^{22}\end{array}$ & Spain & $2003-2013$ & ICD-9 & Minimum Basic Set discharge registry & $\begin{array}{l}\text { Retrospective } \\
\text { observational } \\
\text { study }\end{array}$ & - \\
\hline $\begin{array}{l}\text { Formiga } \\
\text { F } 2018^{39}\end{array}$ & Spain & $2012.1-2014.12$ & $\begin{array}{l}\text { Framingham } \\
\text { criteria }\end{array}$ & Bellvitge University Hospital & - & $>70$ \\
\hline $\begin{array}{l}\text { Golas SB } \\
2018^{23}\end{array}$ & USA & $2014.10 \sim 2015.9$ & ICD-9-CM & PHS & $\begin{array}{l}\text { Retrospective } \\
\text { study }\end{array}$ & $\geq 18$ \\
\hline $\begin{array}{l}\text { Harikrishnan } \\
\text { S } 2017^{40}\end{array}$ & India & $2013-2014$ & $\begin{array}{l}\text { European } \\
\text { Society of HF }\end{array}$ & THFR & - & - \\
\hline $\begin{array}{l}\text { Leong KT } \\
2007^{41}\end{array}$ & Singapore & $\begin{array}{l}2003.11 .10- \\
2004.4 .10\end{array}$ & $\begin{array}{l}\text { Modified } \\
\text { Framingham } \\
\text { criteria }\end{array}$ & Changi General Hospital & $\begin{array}{l}\text { Observational } \\
\text { prospective study }\end{array}$ & - \\
\hline $\begin{array}{l}\text { Mavrea AM } \\
2015^{42}\end{array}$ & Romania & $\begin{array}{l}2013.1 .1- \\
2013.12 .31\end{array}$ & LVEF & Timisoara City Hospital & Prospectively & - \\
\hline
\end{tabular}

(Continued) 
Table I (Continued).

\begin{tabular}{|c|c|c|c|c|c|c|}
\hline $\begin{array}{l}\text { Author } \\
\text { (Year) }\end{array}$ & Country & Study Period & $\begin{array}{l}\text { Method of HF } \\
\text { Diagnosis }\end{array}$ & Data Source & Study Type & $\begin{array}{l}\text { Study } \\
\text { Population }\end{array}$ \\
\hline $\begin{array}{l}\text { McLaren DP } \\
2016^{24}\end{array}$ & USA & $\begin{array}{l}2007.1 .1- \\
2007.12 .31\end{array}$ & ICD-9 & Rochester Medical Center & Retrospective & $\geq 18$ \\
\hline $\begin{array}{l}\text { Mwita JC } \\
2017^{43}\end{array}$ & $\begin{array}{l}\text { South } \\
\text { Africa }\end{array}$ & $20|4.2-20| 5.2$ & - & PMH & $\begin{array}{l}\text { Observational } \\
\text { study }\end{array}$ & $\geq 18$ \\
\hline $\begin{array}{l}\text { Reynolds } \\
\text { K } 2015^{44}\end{array}$ & USA & $2008-2011$ & ICD-9-CM & KPNW, Kaiser Permanente Georgia & $\begin{array}{l}\text { Retrospective } \\
\text { cohort }\end{array}$ & - \\
\hline $\begin{array}{l}\text { Rudiger } \\
\text { A } 2005^{45}\end{array}$ & European & $2001.12-2003.2$ & Physicians & $\begin{array}{l}\text { University Hospital of Zurich, } \\
\text { Helsinki University Central Hospital }\end{array}$ & Prospective study & - \\
\hline $\begin{array}{l}\text { Siirila-Waris } \\
\text { K } 2006^{46}\end{array}$ & England & $\begin{array}{l}2004.2 .2- \\
2004.5 .30\end{array}$ & $\begin{array}{l}\text { ESC AHF } \\
\text { guideline } \\
\text { criteria }\end{array}$ & Hospitals in Finland & $\begin{array}{l}\text { Prospective } \\
\text { multicenter study }\end{array}$ & - \\
\hline $\begin{array}{l}\text { Stampehl } \\
\text { M } 2019^{47}\end{array}$ & USA & $\begin{array}{l}2010.1 .1- \\
2014.12 .31\end{array}$ & ICD-9-CM & Medicare & $\begin{array}{l}\text { Retrospective } \\
\text { study }\end{array}$ & - \\
\hline $\begin{array}{l}\text { Sterling MR } \\
2018^{25}\end{array}$ & USA & $2011-2015$ & - & Vanderbilt University Medical Center & $\begin{array}{l}\text { Prospective } \\
\text { observational } \\
\text { study }\end{array}$ & $\geq 18$ \\
\hline $\begin{array}{l}\text { Tuppin } \\
\text { P } 2013^{26}\end{array}$ & France & 2009 & ICD-I0 & SNIIRAM & - & - \\
\hline $\begin{array}{l}\text { Whittaker BD } \\
2014^{27}\end{array}$ & USA & $\begin{array}{l}2009.7 .1- \\
2010.6 .30\end{array}$ & ICD-9 & Core Measures databases & $\begin{array}{l}\text { Retrospective } \\
\text { cohort study }\end{array}$ & $\geq 18$ \\
\hline $\begin{array}{l}\text { Wiley JF } \\
2017^{28}\end{array}$ & Australia & - & Cardiologist & Multicenter RCT & RCT & $\geq 18$ \\
\hline
\end{tabular}

Abbreviations: ICD-10, Codes of the 10th Revision of the International Statistical Classification of Diseases; DPC, Diagnosis Procedure Combination; ICD-9-CM, International Classification of Diseases-9th Revision-Clinical Modification codes; KorHF, Korean Heart Failure; ICD-9, International Classification of Diseases 9th Revision codes; HCU, Healthcare Utilization; CMS, Centers for Medicare and Medicaid Services; PHS, Partners Healthcare System; HF, Heart Failure; THFR, Trivandrum Heart Failure Registry; LVEF, Left Ventricular Ejection Fraction; PMH, Princess Marina Hospital; KPNW, Kaiser Permanente Northwest; ESC, European Society of Cardiology; AHF, Acute heart failure; SNIIRAM, National Health Insurance Information System; RCT, randomized controlled trial.

Web of Science, and the Cochrane Library. All articles published in English were obtained before May 26, 2019. The Keywords/terms were "Heart Failure", "Patient Readmission" and "Mortality".

\section{Selection Criteria}

Two investigators performed screening of all titles and abstracts in an independent fashion, retrieving and evaluating the retrieved studies based on full texts. In the final analysis, studies selected for inclusion must have provided data for 30-day and/or 1-year readmission or mortality in hospitalized individuals with HF. Studies were excluded for the following reasons: 1) Did not report risk factors or causes; 2) had already subgrouped patients; 3) were cell culture or animal studies; 4) provided no available data; 5) had a small sample size $(<100)$; and 6) was a systematic review, meta-analysis, case report or comments. In the case of patient cohort overlap, studies with the longest followup were included.

\section{Data Extraction and Methodological Quality Assessment}

To facilitate the data extraction process, two researchers generated a standardized form and independently extracted the data. From all eligible articles, the extracted information included the first author, year, country, study period, method of HF diagnosis and data source, study design, study population, sample size, demographic features, 30day and 1-year readmission rates, mortality, and risk factors for readmission and mortality. 
Study quality was evaluated based on the Critical Appraisal of the Health Research Literature ${ }^{29}$ taking into account the sample size, sample design, sampling frame, study and setting, measures, unbiased assessors, response rate and refusers, and prevalence rates. Each item was given a score. A study with a total score below 6 was considered to be of low quality; otherwise, it was considered to be of high quality ( $\geq 6)$.

\section{Statistical Analysis}

The estimated effect sizes for readmission and mortality are expressed as the mean and $95 \%$ confidence interval (CI). The random-effects model was used to pool 30-day and 1-year mortality or readmission rates across studies, as well as the mean age, sex, and comorbidities. ${ }^{30}$ Heterogeneity was assessed by $I^{2}$ statistics. Subgroup analysis was carried out based on the region, study population, and study quality to determine the sources of heterogeneity. The median and interquartile range for age were converted to the mean and standard deviation (SD) as previously proposed. $^{31}$ Inverse funnel plots were generated to visually assess publication bias. STATA/SE 15.1 was utilized for data analysis.

\section{Patient and Public Involvement Statement}

No patients were involved.

\section{Results}

\section{Study Characteristics}

In total, 2691 articles were reviewed for titles/abstracts, and 2609 articles were excluded. The remaining 27 reports were further assessed. The flow diagram of the study selection process is shown in Figure 1. We identified 27 studies included in this meta-analysis that reported 30-day and 1-year readmission data and mortality after $\mathrm{HF}{ }^{18-28,32-47}$ including 1 trial conducted in

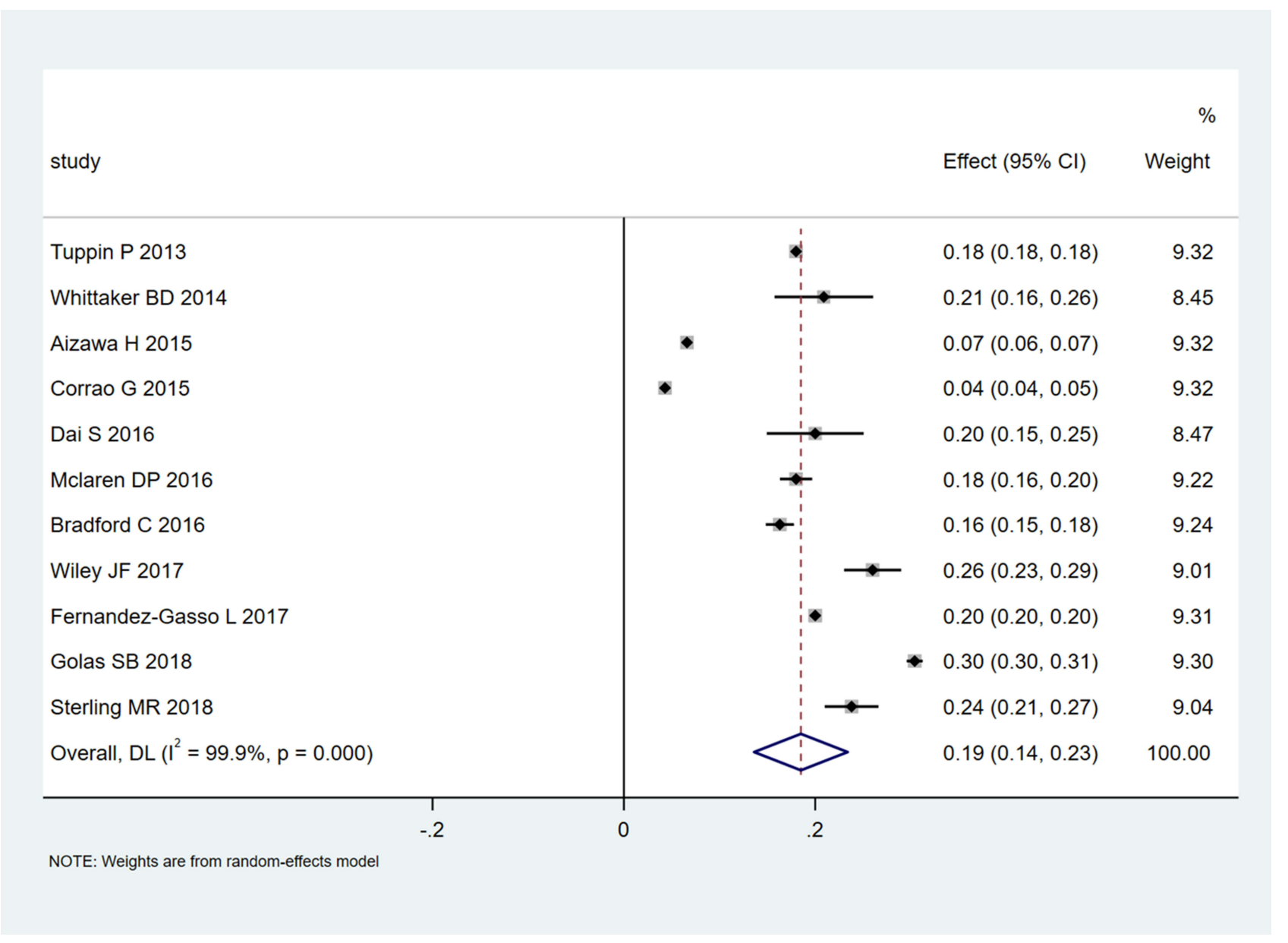

Figure 2 Meta-analysis of 30-day readmission rates. 
Japan, $^{18} 12$ in the US, ${ }^{19,21,23-25,27,33,34,36,38,44,47} 1$ in Italy, ${ }^{20} 2$ in Spain, ${ }^{22,39} 1$ in India, ${ }^{40} 1$ in Singapore, ${ }^{41} 1$ in Romania, ${ }^{42} 1$ in France, ${ }^{26} 1$ in Australia, ${ }^{28} 1$ in Switzerland, $^{32} 1$ in Korea, ${ }^{35} 1$ in Argentina, ${ }^{37} 1$ in South Africa, ${ }^{43} 1$ in Europe ${ }^{45}$ and 1 in England. ${ }^{46}$ The total number of participants was 515,238. Two articles reported both mortality and readmission rates, eleven reported mortality only, and fourteen assessed readmissions only. Ten and four studies were single centre and multicentre trials, respectively, and 13 assessed data from a large national database (Table 1). Twenty-one and 8 studies were of low and high quality, respectively (Tables $\underline{\mathrm{S} 1 \text { and } \mathrm{S} 2}$ ).

\section{0-Day and I-Year Readmission Rates}

A pooled 30-day readmission rate of 0.19 (95\% CI 0.14-0.23; Figure 2) was recorded in 11 studies that included 194,161 patients. Heterogeneity was extremely high $\left(I^{2}=99.9 \%, P<0.001\right)$, and the funnel plot displayed asymmetry. Then, the studies were grouped by region, sample size, and quality for the subgroup analysis (Table S2). The rate was reduced for the nonAmerican region $(0.15,95 \%$ CI $0.08-0.21)$ compared with the American region (0.22, 95\% CI, 0.15-0.28) at 30 days (Table S3). The 30-day readmission rates in studies with sample sizes $<10,000(0.21,95 \%$ CI $0.18-$ 0.24 ) and high quality (score $\geq 6 ; 0.22$, 95\% CI $0.16-$ 0.27) were higher than those for trials with sample sizes $>10,000(0.16,95 \%$ CI $0.09-0.23)$ and low quality (score $<6$; $0.17,95 \%$ CI $0.11-0.23$ ). For 1-year readmissions, the results were similar. The rate was lower for the non-American region $(0.50,95 \%$ CI $0.31-0.68)$ than for the American region $(0.59,95 \%$ CI $0.56-0.62$, Table S4). However, the 1-year admission rate was lower in studies with sample sizes $<10,000(0.49$, 95\% CI $0.30-$ 0.69) compared with those with sample sizes $>10,000$ $(0.59,95 \%$ CI $0.56-0.61)$. Only one study had a quality assessment score above 6 .

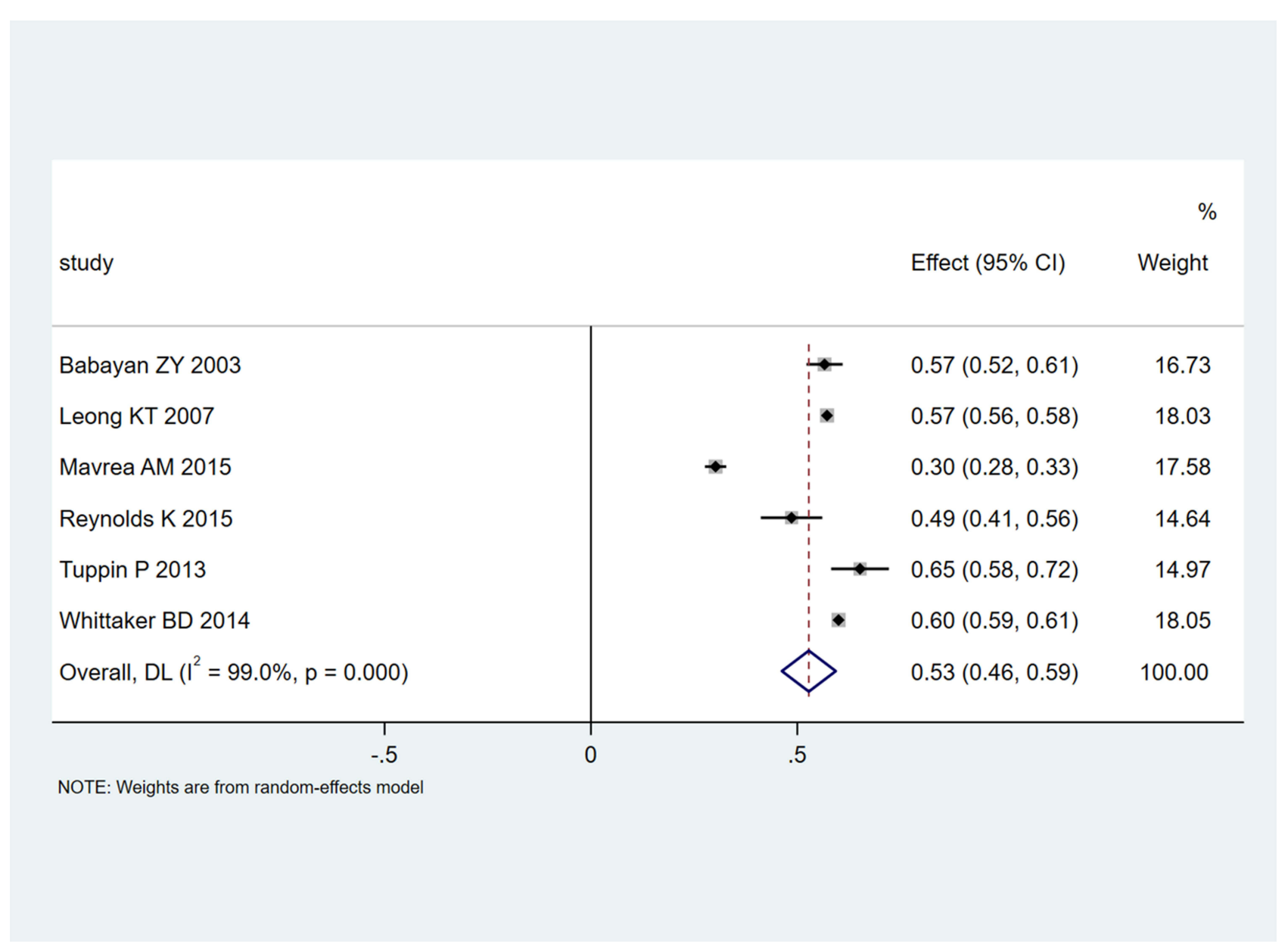

Figure 3 Meta-analysis of I-year readmission rates. 


\begin{tabular}{|c|c|c|c|c|c|c|c|c|c|c|c|c|c|}
\hline 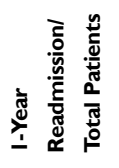 & & 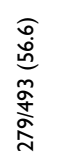 & & 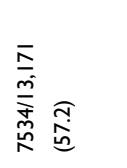 & & & & 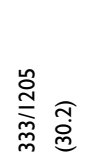 & 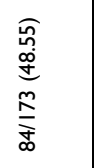 & 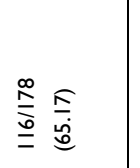 & & 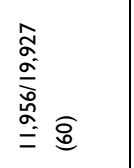 & \\
\hline 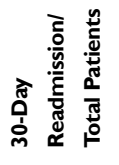 & 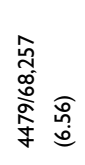 & & 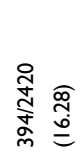 & 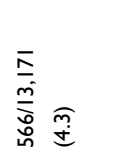 & 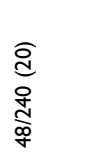 & 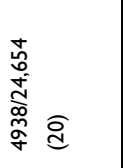 & 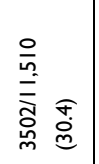 & & & & 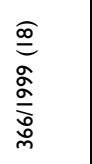 & & 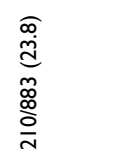 \\
\hline ŏ & 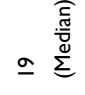 & & & 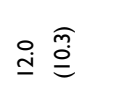 & & & & & & & $\begin{array}{l}+1 \\
\text { ă } \\
\end{array}$ & & \\
\hline 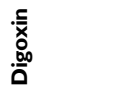 & 吕 & & & & & & & & $\stackrel{\bar{m}}{\bar{\sigma}}$ & & & $\overline{\frac{m}{i}} \overline{\bar{o}}$ & \\
\hline 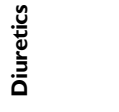 & 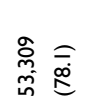 & & & 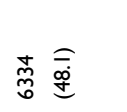 & $\stackrel{\substack{0 \\
\stackrel{\infty}{\infty}}}{\stackrel{\infty}{\infty}}$ & & $\begin{array}{l}\text { o. } \\
\text { o. } \\
\text { ò }\end{array}$ & & 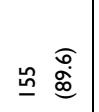 & ฐิ & & 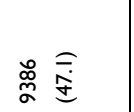 & \\
\hline$\varangle$ & 品 & & & 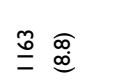 & $\overline{\underline{\underline{\Omega}}}$ 店 & & & & 乃 & ฐิ & & & \\
\hline 粊 & 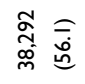 & & & 拿 & 离 & & 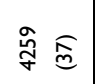 & & 용 $\overline{\bar{s}}$ & & & 旁 & \\
\hline 这 & 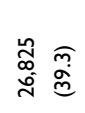 & & & 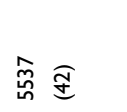 & 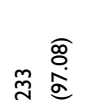 & & 守 $\widehat{\text { qे }}$ & & 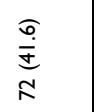 & 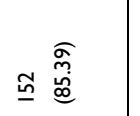 & & 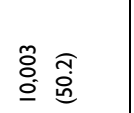 & \\
\hline $\begin{array}{l}\stackrel{0}{\grave{2}} \\
\stackrel{\underline{z}}{\underline{z}} \\
\underline{\underline{I}}\end{array}$ & & & & & 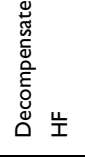 & & & & & $\begin{array}{l}\text { 岀 } \\
\text { 莞 }\end{array}$ & & & \\
\hline$\frac{u}{4}$ & & & & & 学 & & & & & & & & g ํำ \\
\hline نे & & 용 & 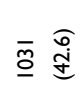 & 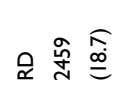 & $\dot{m} \underset{\mathbb{d}}{\mathbb{d}}$ & & & 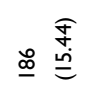 & & $\neq \stackrel{\substack{\mathbb{d} \\
d}}{\stackrel{d}{d}}$ & & & 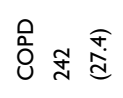 \\
\hline 这 & & ஃ & & 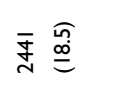 & & & & 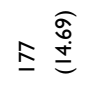 & 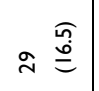 & 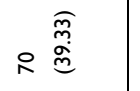 & 产 $\widehat{\alpha}$ & & \\
\hline ชิ & & & 僉宝 & 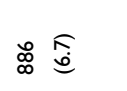 & & & 总 $\overline{\bar{\theta}}$ & 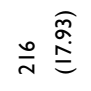 & $\bar{\infty} \stackrel{\substack{\hat{\infty} \\
\dot{f}}}{\mid}$ & 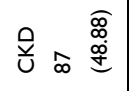 & & 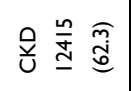 & \\
\hline 로 & & & i $\widehat{d}$ & 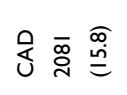 & $\stackrel{\substack{\hat{n} \\
0}}{\underline{\alpha}}$ & & & $\stackrel{\substack{\infty \\
\stackrel{\infty}{\Sigma}}}{\stackrel{c}{\Sigma}}$ & & 号里 & $\stackrel{\infty}{\wedge} \underset{\tilde{c}}{\widehat{c}}$ & 号产字 & 号占 \\
\hline$\frac{z}{I}$ & & 声 & & & 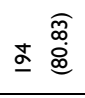 & $\stackrel{\circ}{m} \stackrel{\widehat{\infty}}{\stackrel{D}{=}}$ & 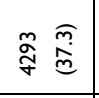 & 今。 & 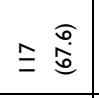 & 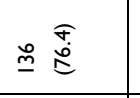 & 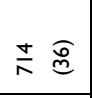 & 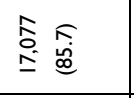 & \\
\hline$\Sigma$ & & g & $\bar{\Sigma} \stackrel{\widehat{a}}{\stackrel{d}{d}}$ & & 拿 & 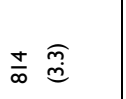 & 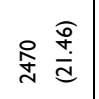 & کु. & $\infty \begin{array}{c}\widehat{\hat{m}} \\
\stackrel{0}{0}\end{array}$ & 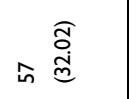 & & 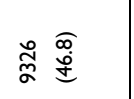 & 命昰 \\
\hline$\frac{\frac{0}{\pi}}{\Sigma}$ & 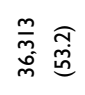 & 完 & 嵒忽 & 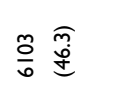 & $\underline{\underline{E}} \widehat{\overline{\mathrm{E}}}$ & 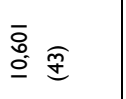 & 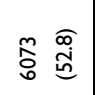 & $\bar{\infty} \underset{\hat{\sigma}}{\widehat{\hat{e}}}$ & $\infty \frac{\widehat{\sigma}}{\frac{5}{n}}$ & 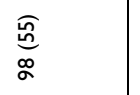 & $\stackrel{\hat{E}}{=}$ 总 & 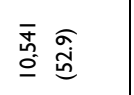 & 安孚 \\
\hline 喿 & & & $\pi$ & ڤू. & & فे & $\hat{n}$ & $\frac{\sim}{i} \stackrel{\kappa}{\stackrel{\tilde{m}}{=}}$ & ô & : & 䓵 & $\stackrel{a}{\kappa}$ & 8 \\
\hline 施 & 胥 & 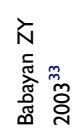 & 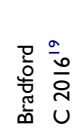 & 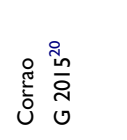 & 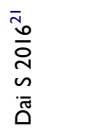 & 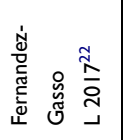 & 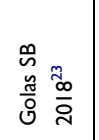 & 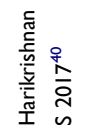 & 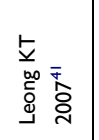 & 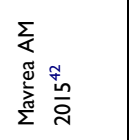 & 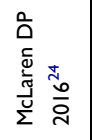 & 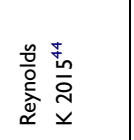 & 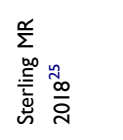 \\
\hline
\end{tabular}




\begin{tabular}{|c|c|c|c|}
\hline 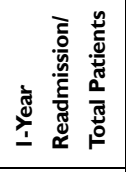 & & & \\
\hline 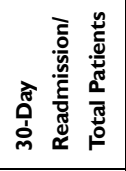 & 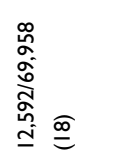 & 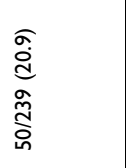 & 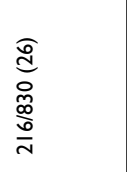 \\
\hline o & $\sigma$ & $\stackrel{+1}{\stackrel{+}{\alpha}} \underset{\dot{I}}{\dot{ \pm}}$ & \\
\hline 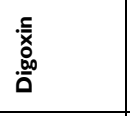 & & & \\
\hline 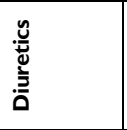 & 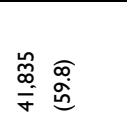 & & \\
\hline$\varangle$ & & & \\
\hline 产 & 号 & 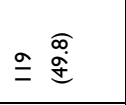 & \\
\hline 总 & 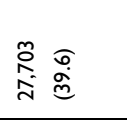 & & \\
\hline $\begin{array}{l}. \\
\stackrel{0}{z} \\
\stackrel{2}{I} \\
\end{array}$ & & & 岌 \\
\hline 岃 & & & \\
\hline ò & & 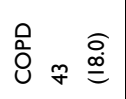 & $\begin{array}{l}\text { 京 } \\
\stackrel{\alpha}{\alpha} \underset{f}{q}\end{array}$ \\
\hline$\stackrel{u}{<}$ & & & \\
\hline uे & & 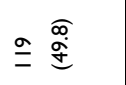 & \\
\hline$\underline{\underline{\underline{I}}}$ & 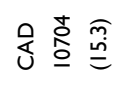 & 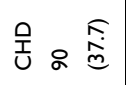 & 足奇 \\
\hline$\underset{I}{z}$ & $\stackrel{\circ}{\circ} \cong$ & 三 & 品 $\bar{E}$ \\
\hline$\Sigma$ & 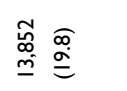 & 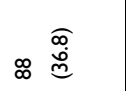 & $\frac{ㅇ ㅡ ㅇ ~}{\overline{0}}$ \\
\hline$\frac{\frac{0}{\pi}}{\Sigma}$ & 总 & 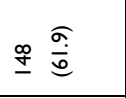 & 昌氛 \\
\hline 迆 & $\stackrel{\infty}{\wedge}$ & 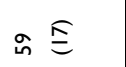 & $\stackrel{\underline{m}}{=}$ \\
\hline 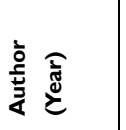 & 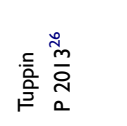 & 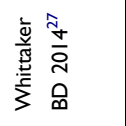 & 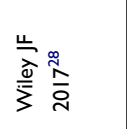 \\
\hline
\end{tabular}

Six studies with 35,147 patients reported 1-year readmission rates. A pooled 1-year readmission rate of 0.53 (95\% CI 0.46-0.59; Figure 3) was obtained, with significant heterogeneity among the trials $\left(I^{2}=99 \%\right.$, $P<0.001)$.

\section{Risk Factors for Readmission}

Fourteen risk factors were revealed by $\geq 2$ trials by multivariate analysis. Common comorbidities, including kidney disease, diabetes, chronic obstructive pulmonary disease (COPD), and cardiac arrhythmia, were tightly associated with elevated 30-day readmission rates. Table 2 depicts all the risk factors for readmission.

\section{0-Day and I-Year Mortality Rates}

A pooled 30-day mortality rate of 0.14 (95\% CI 0.10 0.18 ; Figure 4) in 7 studies that included 317,128 participants was found. Heterogeneity was extremely high $\left(I^{2}=99.9 \%, P<0.001\right)$, and the funnel plot showed asymmetry. The results of the subgroup analyses were not significantly different for 30-day mortality rates (Table S5). It is worth noting that heterogeneity for studies with a sample size $<10,000$ was low $\left(I^{2}=23 \%\right)$. The 1-year mortality rate for the non-American region $(0.28,95 \%$ CI $0.25-0.32)$ was reduced in comparison with the American rate $(0.31,95 \%$ CI $0.31-0.31$; $I^{2}=0 \%$ ) (Table S6).

A pooled 1-year mortality rate of $0.29(95 \%$ CI $0.25-$ 0.33 ; Figure 5) was obtained in 10 studies that included 231,019 participants. Heterogeneity was extremely high $\left(I^{2}=98.8 \%, \quad P<0.001\right)$, and the funnel plot showed asymmetry.

\section{Risk Factors for Mortality}

Fifteen risk factors were revealed by the multivariable analysis in 2 or more trials (Table 3). The risk factors for 30-day mortality included ischaemic heart disease (IHD) in 4 studies. The use of beta-blockers was positively correlated with elevated readmission rates in 3 trials. Meanwhile, a history of lung disease was negatively correlated with readmission in 3 trials.

\section{Heterogeneity Analysis}

The results of this study may be more than expected based on chance alone, with a $P \leq 0.10$ in the heterogeneity test. Given the potential statistical heterogeneity, we formed a hypothesis before conducting the above 
analysis, which may help explain the differences in the results: differences in intervention methods, such as telephone follow-up, home visits, and heart failure clinic visits, may explain the variability leading to the differences in the results.

\section{Discussion}

This was the first comprehensive systematic review and meta-analysis of 30-day and 1-year readmission and mortality rates following HF. Readmission and mortality mostly resulted from cardiac and noncardiac factors. Nonspecific chest pain was the top noncardiac cause of readmission, while cardiac factors encompassed angina and acute ischaemic heart disease, chest pain, etc. In addition, kidney disease, female sex, diabetes mellitus, chronic obstructive pulmonary disease (COPD), and HF were the major predictive factors of early readmission, ${ }^{48}$ which may provide a possible correct direction for reducing the readmission rate of HF patients. Among the 13,171 newly hospitalized HF patients, respiratory disease accounted for $18.7 \%$, arrhythmia accounted for $18.5 \%$, coronary/aortic disease accounted for $15.8 \%$, and renal dysfunction accounted for $6.7 \%{ }^{20}$ The 3 most common reasons for readmission were $\mathrm{HF}$ $(36.0 \%)$, renal disorders $(8.4 \%)$, and other cardiac diseases $(6.9 \%) .{ }^{19}$ In addition, comparing the baseline patients and clinical characteristics of the readmission and nonrehospitalization HF patient groups, COPD and renal disease accounted for the majority of readmissions. ${ }^{19}$

The results indicated that the 30-day readmission rate recorded in 11 studies was 0.19 (95\% CI $0.14-0.23$; Figure 2). Our findings corroborate the data from other nations. For instance, 30-day HF readmission rates after HF hospitalization in the USA and France are both $18 \%{ }^{24,26}$ Meanwhile, the 1-year HF readmission and 30day mortality rates in South Africa are $14.7 \%{ }^{43}$ Most of

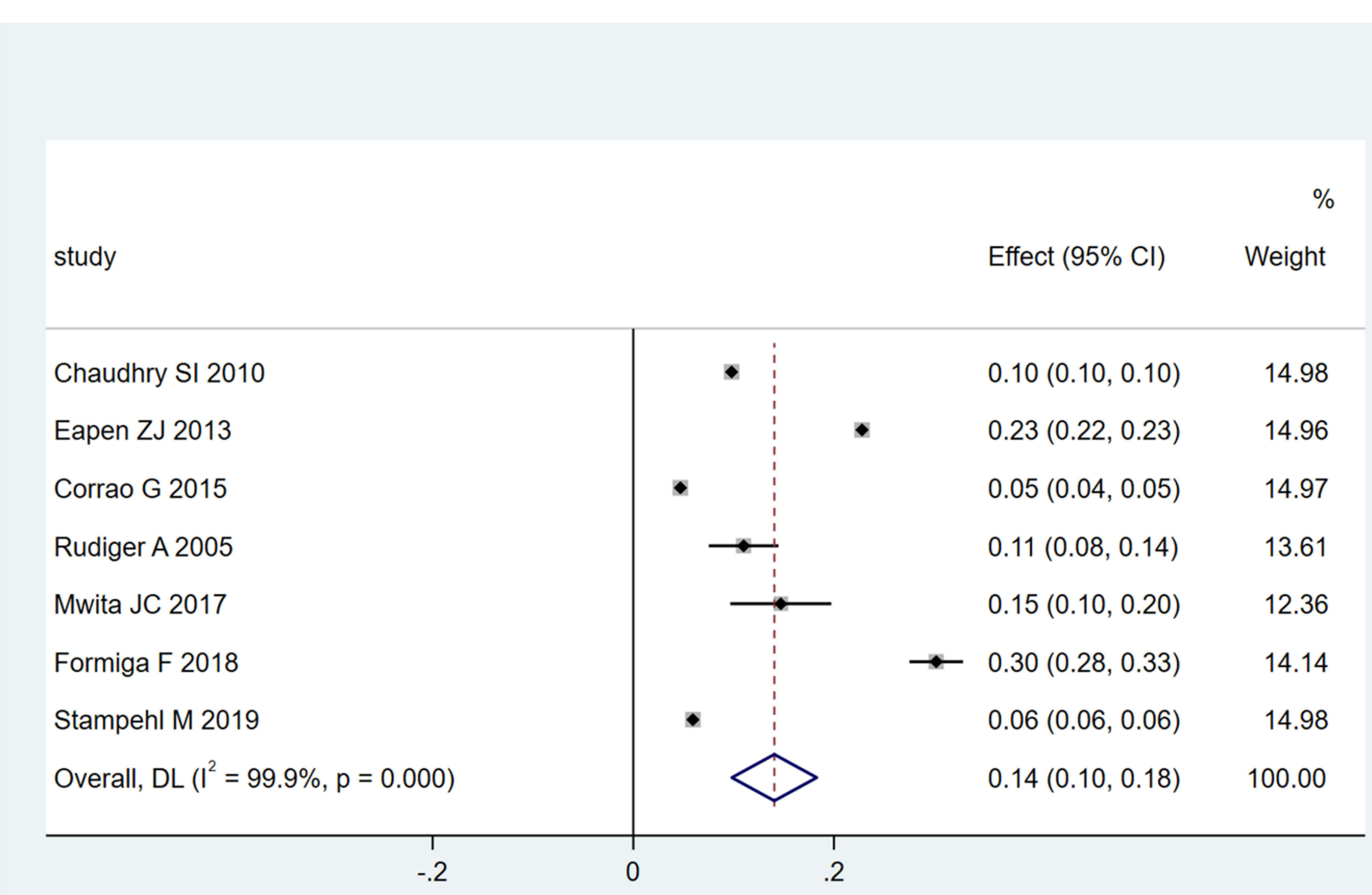

NOTE: Weights are from random-effects model

Figure 4 Meta-analysis of 30-day mortality rates. 
the articles reported similar data for 1-year all-cause mortality, ie, 29\%. ${ }^{32,34,36,38-40,43,45-47}$ The careful management of HF outpatients who are elderly, high disease severity, multiple comorbidities, or taking beta-blockers, loop diuretics, thiazide, or nitrates when discharged from the hospital may be critical to reducing the 30-day readmission. $^{18}$

In some studies assessed in this meta-analysis, the authors identified multiple predictive factors of readmission, including age $\mathrm{a}^{18,20,21}$ and clinical comorbidities. $^{20,33,40-42}$ Some reports revealed multiple parameters that increased 30-day readmission rates, including elevated New York Heart Association functional class (NYHA) and Charlson Comorbidity Index (CCI) and treatment with beta-blockers, loop diuretics, thiazide, or nitrates. ${ }^{18}$ In addition, retired and/or disabled patients had one or more emergency room visits in the last 3 months, hospitalization durations above 5 days, and BUN levels $>45 \mathrm{mg} / \mathrm{dL}$ at discharge. ${ }^{19}$ However, only one study reported that age, sex, race, marital status, payer type, and multiple patient features did not predict readmission in their model from Bradford et al. ${ }^{19}$

This study had limitations. First, due to limited data, the original causes or comorbidities of $\mathrm{CHF}$ patients are not yet clear. Second, multiple risk factors for and/or causes of readmission had no clear definitions, and various reports classified and grouped the causes and risk factors differently with variable definitions of the parameters, which were hardly combined for the meta-analysis. Finally, the studies were highly heterogeneous. Most reports had incomplete datasets, and subgroup analyses could not be performed for all variables.

\section{Conclusions}

We found that multiple diseases are very common in hospitalized chronic HF patients. In addition, the increase in recurrent diseases itself was shown to be parallel to an elevated all-cause 30-day readmission

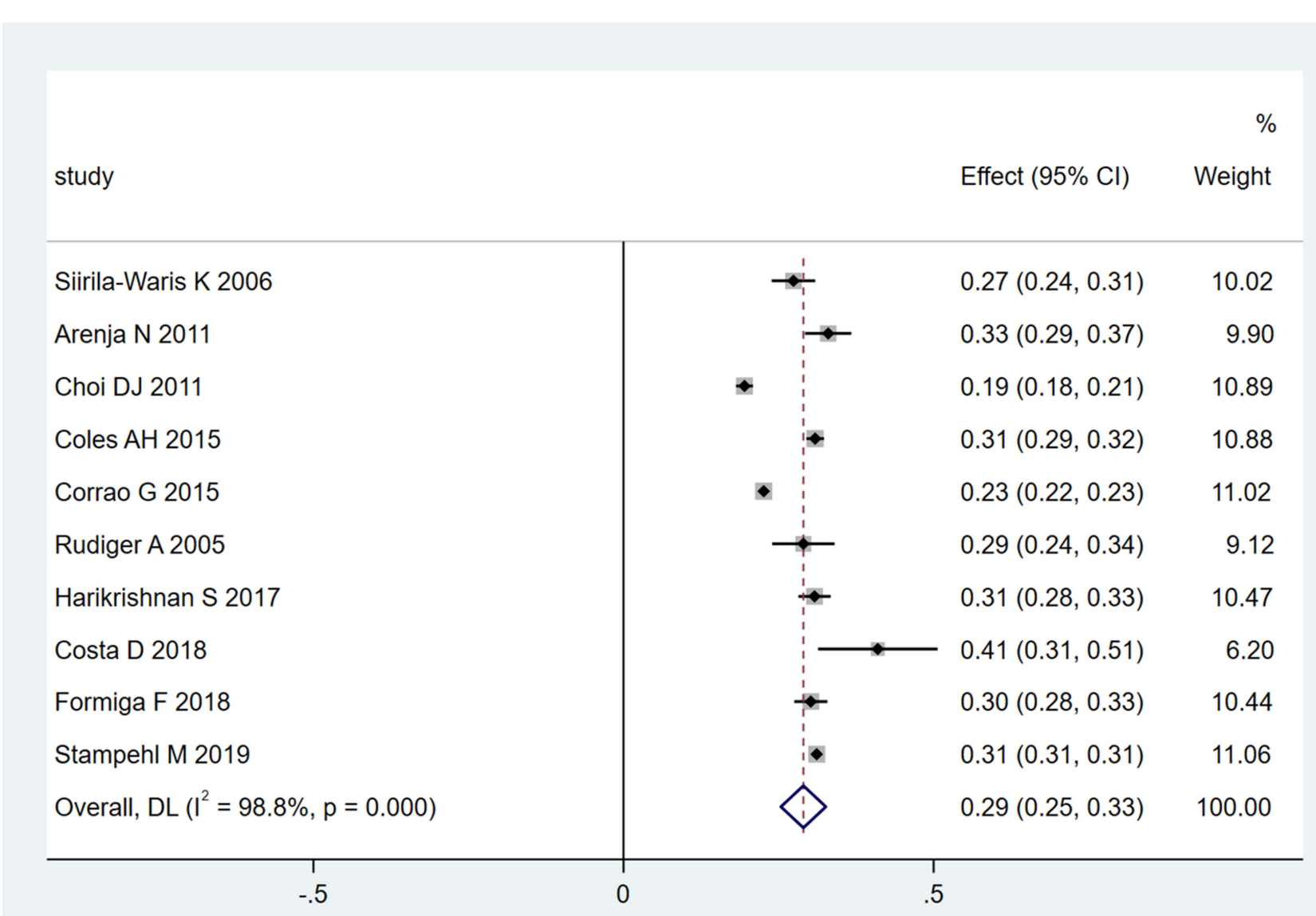

NOTE: Weights are from random-effects model

Figure 5 Meta-analysis of I-year mortality rates. 


\begin{tabular}{|c|c|c|c|c|c|c|c|c|c|c|c|c|}
\hline 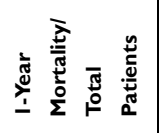 & 产 & , & 总 & 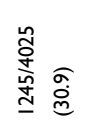 & 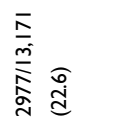 & $\begin{array}{l}\frac{\bar{y}}{8} \\
\frac{8}{\frac{8}{7}}\end{array}$ & , & 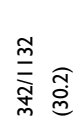 & 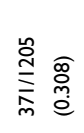 & 1 & 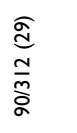 & 产 \\
\hline 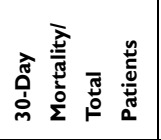 & , & 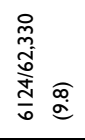 & 1 & 1 & 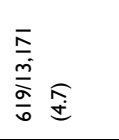 & 1 & 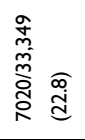 & 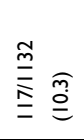 & 1 & 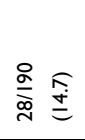 & 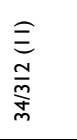 & । \\
\hline ò & 志 烏 & 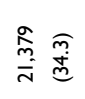 & 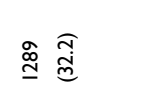 & $\stackrel{\varrho}{\sigma} \stackrel{\varrho}{=}$ & 1 & 1 & , & 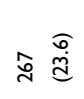 & $\stackrel{\stackrel{\circ}{ }}{\underline{-n}}$ & & , & $\stackrel{\circ}{\underline{I}}$ \\
\hline oิ & I & 1 & 1 & 1 & $\stackrel{\text { 용 }}{\stackrel{\widehat{O}}{=}}$ & I & 1 & I & 1 & 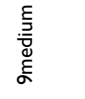 & , & 1 \\
\hline 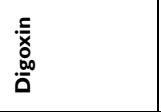 & 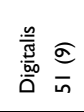 & । & 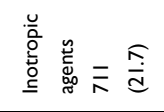 & 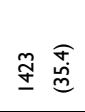 & 1 & $\stackrel{\circ}{\stackrel{\circ}{ }}$ & 1 & 1 & 1 & $\underset{\substack{\bar{d} \\
\infty}}{\infty}$ & । & , \\
\hline 营 & $\begin{array}{l}\widehat{\widetilde{\alpha}} \\
\stackrel{\tilde{g}}{\xi}\end{array}$ & । & 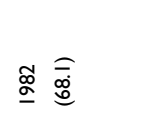 & $\bar{\partial}$ & 誉 & 1 & 1 & I & 1 & 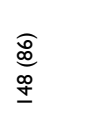 & , & , \\
\hline$\varangle$ & 1 & 1 & $\frac{m}{\sigma} \overline{\bar{p}}$ & 虽 & $\stackrel{\oplus}{=} \underset{\infty}{\stackrel{\infty}{\infty}}$ & ஓे & 1 & $\underline{ \pm} \stackrel{\widehat{\rho}}{=}$ & 1 & . & , & , \\
\hline 严 & 果 & , & 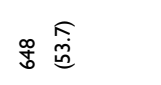 & 奈 & 嗍 & ڤํ & 1 & $\stackrel{\widehat{\infty}}{\stackrel{\alpha}{n}}$ & 1 & 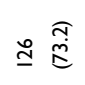 & , & , \\
\hline لَّ & $\begin{array}{l}\widehat{\overline{\hat{\theta}}} \\
\hat{\tilde{m}}\end{array}$ & , & 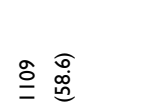 & 尽 & 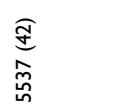 & 今̊ㅇํ & 1 & 总 & 1 & $\pm \widehat{\bar{d}}$ & , & , \\
\hline 岂 & 㞺 & , & । & 榬 & , & 愛 & , & 愛 & , & 㞺 & 愛 & , \\
\hline 出 & 1 & 1 & 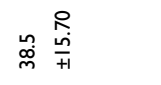 & 1 & 1 & I & 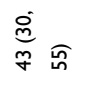 & । & 1 & $\stackrel{\infty}{\vec{\sigma}} \underset{\mathrm{d}}{ }$ & , & , \\
\hline ชั & 1 & 1 & 醇 & 1 & 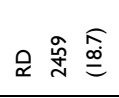 & 1 & 1 & 1 & 1 & 1 & I & 1 \\
\hline$\stackrel{4}{\alpha}$ & 1 & 1 & , & 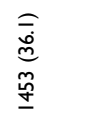 & 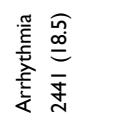 & 户े & $\begin{array}{l}\overline{\bar{\alpha}} \widehat{\overline{\dot{\omega}}} \\
\stackrel{=}{=}\end{array}$ & $\begin{array}{l}\text { a } \\
\text { ô } \\
\text { 市 }\end{array}$ & $\underline{\mathbf{I}}$ & $\begin{array}{l}\widehat{\widehat{\alpha}} \\
\stackrel{\alpha}{\sigma} \\
\stackrel{\sigma}{\sigma}\end{array}$ & $\begin{array}{l}\widehat{a} \\
\stackrel{d}{d} \\
\bar{a}\end{array}$ & ঐे. \\
\hline$\stackrel{\vartheta}{u}$ & 方 $\widehat{\sigma}$ & , & $\stackrel{\mathscr{a}}{\sim} \stackrel{\widehat{a}}{g}$ & 흥 & $\stackrel{\infty}{\underset{\infty}{\mathscr{e}}}$ & , & , & $\stackrel{\infty}{\stackrel{\widehat{N}}{\stackrel{d}{d}}}$ & $\stackrel{\circ}{\sim}$ & , & , & $\stackrel{+}{\sigma}$ \\
\hline 呈 & 1 & 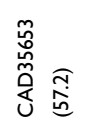 & 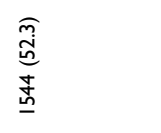 & 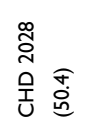 & 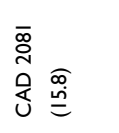 & , & 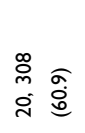 & 迅 & ळ & $\begin{array}{l}\underset{\mathfrak{b}}{\hat{\omega}} \\
= \\
=\end{array}$ & $\underset{\substack{a \\
\infty}}{\stackrel{0}{\infty}}$ & 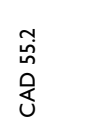 \\
\hline$\underset{I}{z}$ & $\stackrel{8}{q} \widehat{\Xi}$ & 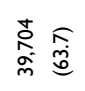 & 榇 & 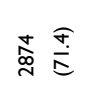 & , & 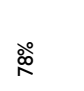 & $\begin{array}{l}\stackrel{M}{0} \widehat{\widehat{m}} \\
\dot{d}\end{array}$ & 文 & 。 & 옹 & & f \\
\hline$\Sigma$ & $\underline{\mathrm{a}}$ 命 & 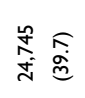 & 合 命 & $\underset{\underline{q}}{\underline{q}} \widehat{\overline{\hat{m}}}$ & 1 & $\stackrel{\circ}{\circ}$ & 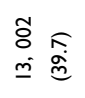 & 字㔬 & క్ర & 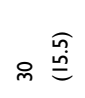 & 으 $\overline{\overline{\tilde{m}}}$ & 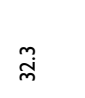 \\
\hline$\frac{\frac{0}{\pi}}{\Sigma}$ & 品 居 & 参 & 兽兑 & 导 & 哭 & $\begin{array}{l}\text { b } \\
\stackrel{6}{n} \\
\text { in }\end{array}$ & $\begin{array}{l}\bar{z} \widehat{\sigma} \\
\underline{\underline{G}}\end{array}$ & 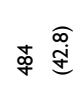 & $\overline{\bar{\infty}} \underset{\underline{a}}{\widehat{\sigma}}$ & 高 & 气̊ & $\simeq$ 楚 \\
\hline$\underset{8}{\stackrel{\circ}{\alpha}}$ & 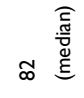 & 옹 & $\stackrel{\circ}{\grave{b}} \stackrel{\widehat{P}}{=}$ & $n$ & 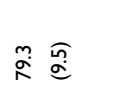 & 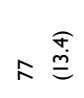 & 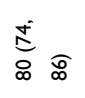 & $\stackrel{\circ}{\infty}$ & 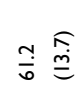 & 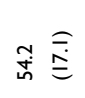 & 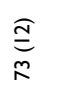 & 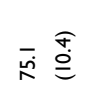 \\
\hline 毫 & 莺 & 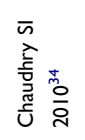 & 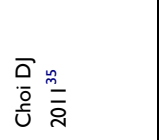 & 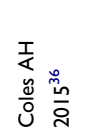 & 密 & 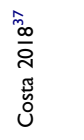 & 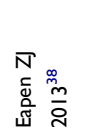 & 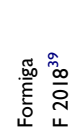 & 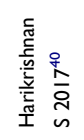 & 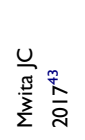 & 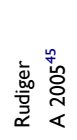 & 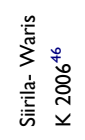 \\
\hline
\end{tabular}




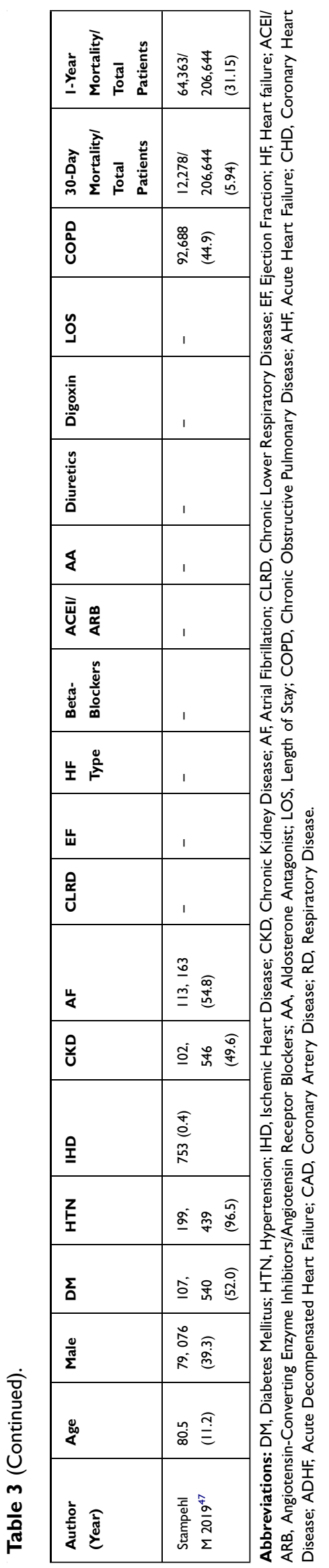

rate. This is a major problem for individuals and the health care system as a whole. Based on the present evidence of common comorbid disease clusters in chronic HF, the development and testing of new interventions tailored to patients in each cluster may be a key direction for future clinical trials.

\section{Data Sharing Statement}

No additional data available.

\section{Acknowledgments}

Our work was funded by fundamental research funds for the National Center for Clinical Medicine of Digestive Diseases (No. 2015BAI13B07, to Dai-Ming Fan).

\section{Disclosure}

The authors declare that they have no conflict of interest.

\section{References}

1. Heidenreich PA, Albert NM, Allen LA, et al. Forecasting the impact of heart failure in the United States: a policy statement from the American Heart Association. Circulation. 2013;6(3):606-619. doi:10.1161/HHF.0b013e318291329a

2. Colby SL, Ortman JM. Projections of the Size and Composition of the U.S. Population 2014 to 2060. U.S. Department of Commerce: Economics and Statistics Administration: U.S. Census Bureau; 2014.

3. Heidenreich PA, Trogdon JG, Khavjou OA, et al. Forecasting the future of cardiovascular disease in the United States: a policy statement from the American Heart Association. Circulation. 2011;123:933-944. doi:10.1161/CIR.0b013e31820a55f5

4. Finkelstein EA, Khavjou OA, Thompson H, et al. Medicine, obesity and severe obesity forecasts through 2030. Am J Prev Med. 2012;42 (6):563-570. doi:10.1016/j.amepre.2011.10.026

5. Boyle JP, Thompson TJ, Gregg EW, Barker LE, Williamson DF. Projection of the year 2050 burden of diabetes in the US adult population: dynamic modeling of incidence, mortality, and prediabetes prevalence. Popul Health Metr. 2010;8(1):29. doi:10.1186/ 1478-7954-8-29

6. Yancy CW, Jessup M, Bozkurt B, et al. 2013 ACCF/AHA guideline for the management of heart failure: a report of the American college of cardiology foundation/American heart association task force on practice guidelines. $J \mathrm{Am}$ Coll Cardiol. 2013;62:e147-e239. doi:10.1016/j.jacc.2013.05.019

7. Levy D, Kenchaiah S, Larson MG, et al. Long-term trends in the incidence of and survival with heart failure. $N$ Engl $J$ Med. 2002;347:1397-1402. doi:10.1056/NEJMoa020265

8. Stewart S, Macintyre K, Hole DJ, Capewell S, McMurray JJV. More 'malignant' than cancer? Five-year survival following a first admission for heart failure. Eur J Heart Fail. 2001;3(3):315-322. doi:10.1016/S1388-9842(00)00141-0

9. Abhayaratna WP, Smith WT, Becker NG, Marwick TH, Jeffery IM, Mcgill DA. Prevalence of heart failure and systolic ventricular dysfunction in older Australians: the Canberra Heart Study. Med J Aust. 2006; 184:151-154.

10. Sahle BW, Owen AJ, Mutowo MP, Krum H, Reid CM. Prevalence of heart failure in Australia: a systematic review. BMC Cardiovasc Disord. 2016;16(1):32. doi:10.1186/s12872-016-0208-4 
11. Jencks SF, Williams MV, Coleman EA. Rehospitalizations among patients in the medicare fee-for-service program. J Vasc Surg. 2009;50(1):234. doi:10.1016/j.jvs.2009.05.045

12. Giamouzis G, Kalogeropoulos A, Georgiopoulou V, et al. Hospitalization epidemic in patients with heart failure: risk factors, risk prediction, knowledge gaps, and future directions. J Card Fail. 2011;17(1):0-75. doi:10.1016/j.cardfail.2010.08.010

13. Ambrosy AP, Fonarow GC, Butler J, Chioncel O. The global health and economic burden of hospitalizations for heart failure lessons learned from hospitalized heart failure registries. $J \mathrm{Am}$ Coll Cardiol. 2014;63:1123-1133. doi:10.1016/j.jacc.2013.11.053

14. Dharmarajan K, Wang Y, Lin Zet al. Association of changing hospital readmission rates with mortality rates after hospital discharge. JAMA. 2017;318:270-278. doi:10.1001/jama.2017.8444

15. Chen HY, Tisminetzky M, Lapane KL, et al. Disease, decade-long trends in 30-day rehospitalization rates after acute myocardial infarction. J Am Heart Assoc. 2015;4:e002291. doi:10.1161/JAHA.115. 002291

16. Amarasingham R, Patel PC, Toto K, et al. Allocating scarce resources in real-time to reduce heart failure readmissions: a prospective, controlled study. BMJ Qual Saf. 2013;22(12):998-1005. doi:10.1136/ bmjqs-2013-001901

17. Amarasingham R, Moore BJ, Tabak YP, et al. An automated model to identify heart failure patients at risk for 30-day readmission or death using electronic medical record data. Med Care. 2010;48:981-988.

18. Aizawa H, Imai S, Fushimi K. Factors associated with 30-day readmission of patients with heart failure from a Japanese administrative database. BMC Cardiovasc Disord. 2015;15(1):134. doi:10.1186/ s12872-015-0127-9

19. Bradford C, Shah BM, Shane P, Wachi N, Sahota K. Patient and clinical characteristics that heighten risk for heart failure readmission. Res Social Adm Pharm. 2017;13(6):1070-1081. doi:10.1016/j. sapharm.2016.11.002

20. Corrao G, Ghirardi A, Ibrahim B, Merlino L, Maggioni AP. Shortand long-term mortality and hospital readmissions among patients with new hospitalization for heart failure: a population-based investigation from Italy. Int J Cardiol. 2015;181:81-87. doi:10.1016/j. ijcard.2014.12.004

21. Dai S, Manoucheri M, Gui J, et al. Kansas City cardiomyopathy questionnaire utility in prediction of 30-day readmission rate in patients with chronic heart failure. Cardiol Res Pract. 2016;20 16:4571201. doi:10.1155/2016/4571201

22. Fernandez-Gasso L, Hernando-Arizaleta L, Palomar-Rodriguez JA, Abellan-Perez MV, Pascual-Figal DA. Trends, causes and timing of 30-day readmissions after hospitalization for heart failure: 11-year population-based analysis with linked data. Int $J$ Cardiol. 2017;248:246-251. doi:10.1016/j.ijcard.2017.07.094

23. Golas SB, Shibahara T, Agboola S, et al. A machine learning model to predict the risk of 30-day readmissions in patients with heart failure: a retrospective analysis of electronic medical records data. BMC Med Inform Decis Mak. 2018;18(1):44. doi:10.1186/s12911018-0620-z

24. McLaren DP, Jones R, Plotnik R, et al. Prior hospital admission predicts thirty-day hospital readmission for heart failure patients. Cardiol J. 2016;23(2):155-162. doi:10.5603/CJ.a2016.0005

25. Sterling MR, Safford MM, Goggins K, et al. Numeracy, health literacy, cognition, and 30-day readmissions among patients with heart failure. J Hosp Med. 2018;13:145-151. doi:10.12788/jhm.2932

26. Tuppin P, Cuerq A, de Peretti C, et al. First hospitalization for heart failure in France in 2009: patient characteristics and 30-day follow-up. Arch Cardiovasc Dis. 2013;106:570-585. doi:10.1016/j. acvd.2013.08.002

27. Whittaker BD, Soine LA, Errico KM. Patient and process factors associated with all-cause 30-day readmission among patients with heart failure. J Am Assoc Nurse Pract. 2015;27:105-113. doi:10. 1002/2327-6924.12123
28. Wiley JF, Chan YK, Ahamed Y, et al. Multimorbidity and the risk of all-cause 30-day readmission in the setting of multidisciplinary management of chronic heart failure: a retrospective analysis of 830 hospitalized patients in Australia. $J$ Cardiovasc Nurs. 2018;33:437-445. doi:10.1097/JCN.0000000000000391

29. Loney PL, Chambers LW, Bennett KJ, Roberts JG. Critical appraisal of the health research literature: prevalence or incidence of a health problem. Chronic Dis Can. 1998;19:170-176.

30. Nyaga VN, Arbyn M. Metaprop: a stata command to perform meta-analysis of binomial data. Arch Public Health. 2014;72:39. doi:10.1186/2049-3258-72-39

31. Wan X, Wang W, Liu J, Tong T. Estimating the sample mean and standard deviation from the sample size, median, range and/or interquartile range. BMC Med Res Methodol. 2014;14(1):1-3. doi:10.1186/1471-2288-14-135

32. Arenja N, Breidthardt T, Socrates T, et al. Risk stratification for 1-year mortality in acute heart failure: classification and regression tree analysis. Swiss Med Wkly. 2011;141:w13259. doi:10.4414/ smw.2011.13259

33. Babayan ZV, McNamara RL, Nagajothi N, et al. Predictors of cause-specific hospital readmission in patients with heart failure. Clin Cardiol. 2003;26:411-418. doi:10.1002/clc.4960260906

34. Chaudhry SI, Wang Y, Gill TM, Krumholz HM. Geriatric conditions and subsequent mortality in older patients with heart failure. $J \mathrm{Am}$ Coll Cardiol. 2010;55:309-316. doi:10.1016/j.jacc.2009.07.066

35. Choi DJ, Han S, Jeon ES, et al. Characteristics, outcomes and predictors of long-term mortality for patients hospitalized for acute heart failure: a report from the Korean heart failure registry. Korean Circ J. 2011;41:363-371. doi:10.4070/kcj.2011.41.7.363

36. Coles AH, Tisminetzky M, Yarzebski J, et al. Magnitude of and prognostic factors associated with 1-year mortality after hospital discharge for acute decompensated heart failure based on ejection fraction findings. $J$ Am Heart Assoc. 2015;4. doi:10.1161/ JAHA. 115.002303

37. Costa D, Aladio M, Girado CA, de la Hoz RP, Berensztein CS. Frailty is independently associated with 1-year mortality after hospitalization for acute heart failure. Int $J$ Cardiol Heart Vasc. 2018;21:103-106. doi:10.1016/j.ijcha.2018.10.004

38. Eapen ZJ, Liang L, Fonarow GC, et al. Validated, electronic health record deployable prediction models for assessing patient risk of 30-day rehospitalization and mortality in older heart failure patients. JACC Heart Fail. 2013;1:245-251. doi:10.1016/j.jchf.2013.01.008

39. Formiga F, Moreno-Gonzalez R, Chivite D, et al. Sex differences in 1-year mortality risks in older patients experiencing a first acute heart failure hospitalization. Geriatr Gerontol Int. 2019;19:184-188.

40. Harikrishnan S, Sanjay G, Agarwal A, et al. One-year mortality outcomes and hospital readmissions of patients admitted with acute heart failure: data from the trivandrum heart failure registry in Kerala, India. Am Heart J. 2017;189:193-199. doi:10.1016/j. ahj.2017.03.019

41. Leong KT, Goh PP, Chang BC, Lingamanaicker J. Heart failure cohort in Singapore with defined criteria: clinical characteristics and prognosis in a multi-ethnic hospital-based cohort in Singapore. Singapore Med J. 2007;48:408-414.

42. Mavrea AM, Dragomir T, Bordejevic DA, Tomescu MC, Ancusa O, Marincu I. Causes and predictors of hospital readmissions in patients older than 65 years hospitalized for heart failure with preserved left ventricular ejection fraction in western Romania. Clin Interv Aging. 2015;10:979-990. doi:10.2147/CIA.S83750

43. Mwita JC, Dewhurst MJ, Magafu MG, et al. Presentation and mortality of patients hospitalised with acute heart failure in Botswana. Cardiovasc J Afr. 2017;28:112-117. doi:10.5830/CVJA-2016-067

44. Reynolds K, Butler MG, Kimes TM, Rosales AG, Chan W, Nichols GA. Relation of acute heart failure hospital length of stay to subsequent readmission and all-cause mortality. Am J Cardiol. 2015;116:400-405. doi:10.1016/j.amjcard.2015.04.052 
45. Rudiger A, Harjola VP, Muller A, et al. Acute heart failure: clinical presentation, one-year mortality and prognostic factors. Eur J Heart Fail. 2005;7:662-670. doi:10.1016/j.ejheart.2005. 01.014

46. Siirila-Waris K, Lassus J, Melin J, Peuhkurinen K, Nieminen MS, Harjola VP. Characteristics, outcomes, and predictors of 1-year mortality in patients hospitalized for acute heart failure. Eur Heart J. 2006;27:3011-3017. doi:10.1093/eurheartj/ehl407
47. Stampehl M, Friedman HS, Navaratnam P, Russo P, Park S, Obi EN. Risk assessment of post-discharge mortality among recently hospitalized medicare heart failure patients with reduced or preserved ejection fraction. Curr Med Res Opin. 2019;36(2):179-188.

48. Wang H, Zhao T, Wei X, Lu H, Lin X. The prevalence of 30-day readmission after acute myocardial infarction: a systematic review and meta-analysis. Clin Cardiol. 2019;42(10):889-898. doi:10.1002/ clc. 23238

\section{Publish your work in this journal}

Therapeutics and Clinical Risk Management is an international, peerreviewed journal of clinical therapeutics and risk management, focusing on concise rapid reporting of clinical studies in all therapeutic areas, outcomes, safety, and programs for the effective, safe, and sustained use of medicines. This journal is indexed on PubMed Central, CAS,
EMBase, Scopus and the Elsevier Bibliographic databases. The manuscript management system is completely online and includes a very quick and fair peer-review system, which is all easy to use. Visit http://www.dovepress.com/testimonials.php to read real quotes from published authors. 